\title{
Improved Content Based Medical Image Retrieval using PCA with SURF Features
}

\author{
S.Govindaraju, B.Mukunthan
}

\begin{abstract}
In the computer era, the Content Based Image Retrieval system (CBIR) has most widely used in medical field and crime invention. During the last decade, CBIR emerged as powerful tool to efficiently retrieved images visually similar to query image. The basic process behind this concept is representation of image as feature vector and to measure the similarities between the images with distance between their corresponding feature vectors according to some metrics. The finding of correct features to represent images with, as well as the similarity metric that groups visually similar image together, are important milestone in construction of any CBIR system .The work in this paper focused on retrieve the correct query image from a huge number of medical image databases with the help of Principal Component Analysis (PCA) through SURF feature vector detection. The combination of this method produces an accurate and quick response than other conventional methods like SIFT and SURF feature vector based medical image retrieval.
\end{abstract}

Index Terms: CBMIR, SURF, PCA, MRI Images, Fast-Hessian matrix.

\section{INTRODUCTION}

In the medical world, medical image retrieval is one of the challenging tasks. Since that all the medical images have been captured in the form of black and white or gray level using this values we could not predict correct diseases. In the advent of current years, content-based image retrieval has advanced remarkably in the field of computer vision such as medical imaging, geographical information, crime prevention,

education and training, personal photos, and etc, [1]. Retrieval of images in the CBIR system is based on the various visual features. The first version of CBIR system was based on the query by image and measures the similarity for retrieving the images from database [3].

This work attempts to introduce some additional information in the retrieval process by the use of a feature detection method. There is a two stage implementation is performed in this paper. First detect image local features with

Revised Manuscript Received on July 08, 2019.

S.Govindaraju, Assistant Professor (Part-Time Ph.D., Research Scholar), Department of Computer Science, School of Computing, Sri Ramakrishna College of Arts \& Science (formerly SNR Sons College), Bharathiar University, Coimbatore-641006, Tamil Nadu, India.

Dr B.Mukunthan, Assistant Professor, Department of Computer Science, School of Computing, Sri Ramakrishna College of Arts \& Science (formerly SNR Sons College), Bharathiar University, Coimbatore-641006, Tamil Nadu, India.
SURF and then convince the dimensionality of feature vector with PCA. The combination of PCA with SURF gives acceptable result than other existing methods.

This paper is organized as unit-I give a detailed introduction about CBIR system, the existing related works have been described in section-II, section III defines a research methodology. The Experiments and results are discussed in Unit IV and Unit $\mathrm{V}$ gives a conclusion about this work.

\section{RELATED WORK}

K.Somasundaram et al, the proposed methods consist of two stage brain extraction algorithm to extract the brain from T2-weighted MRI images. We have developed two brain extraction algorithms 2D-BEA and 3D-BEA to extract brain portion from T2-weighted MRI head scans automatically. Both of them do not require any initial parameters that are to be supplied by the user. R.Rajesh et al, the linear index of fuzziness for type-1 fuzzy sets by Zenzo et al and measure of ultra fuzziness for type-2 fuzzy sets by Tizhoosh has difficulties in handling MRI brain images with one level of gray value as background and other two levels of grayness as white matter and gray matter. Hence this paper proposes new modified thresholding measures for MRI brain images using type1 and type-2 fuzzy sets. The results show the effectiveness of the proposed modified thresholding measures. Due to the difficulties in handling MRI brain images with one level of gray value as background and other two levels of grayness as white matter and gray matter, the paper has presented modified index of fuzziness for type 1 fuzzy sets and modified ultra fuzziness for type 2 fuzzy sets for thresholding brain images. E. Ben George et al, this paper proposes a novel approach for the MRI image enhancement, which is based on the Modified Tracking Algorithm, Histogram Equalization and Center Weighted Median (CWM) filter. This method consists of two approaches. The first approach is applying the modified tracking algorithm to remove the film artifacts, labels and skull region and then applying the Histogram Equalization and Center Weighted Median (CWM) filter techniques separately to enhance the images. D.Napoleon et al, in the proposed method an efficient detection of brain tumor region from cerebral image is done using Kernel Induced Possiblistic C-means clustering and histogram. The using Kernel Induced Possiblistic C-means clustering algorithm finds the centroids of the cluster groups together the Brain tumor patterns obtained from MRI images.

The accurate results of Kernel

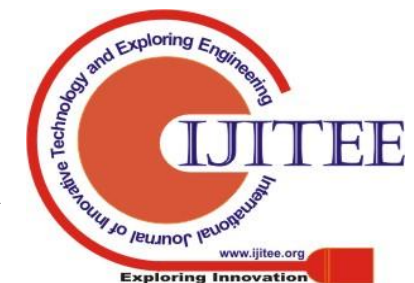


Induced Fuzzy C-means clustering algorithm effectively extract the tumor region from brain MRI brain images.

\section{RESEARCH METHODOLOGY}

The image retrival process contains two levels of process, First level perform the preprocessing and then retrieve the image based on their content. The important work of the preprocessing level is feature extraction. This can be performed by SURF feature detector. The PCA approach is used for classification. Various researches have been done on PCA algorithm that provides more accurate image classifier system than other techniques [3]. In CBIR systems the word "Content-based" means, the search searches the contents of image rather than metadata such as tags, keywords and descriptions related with the image. The CBIR system retrieves the image from digital image database on the basis of SURF features. When a query image is input in CBIR system, The image features extracted and matching with classification is performed between the testing image and image database features. The results were for requested testing image is then retrieved from the database which is foremost closes to the query image.

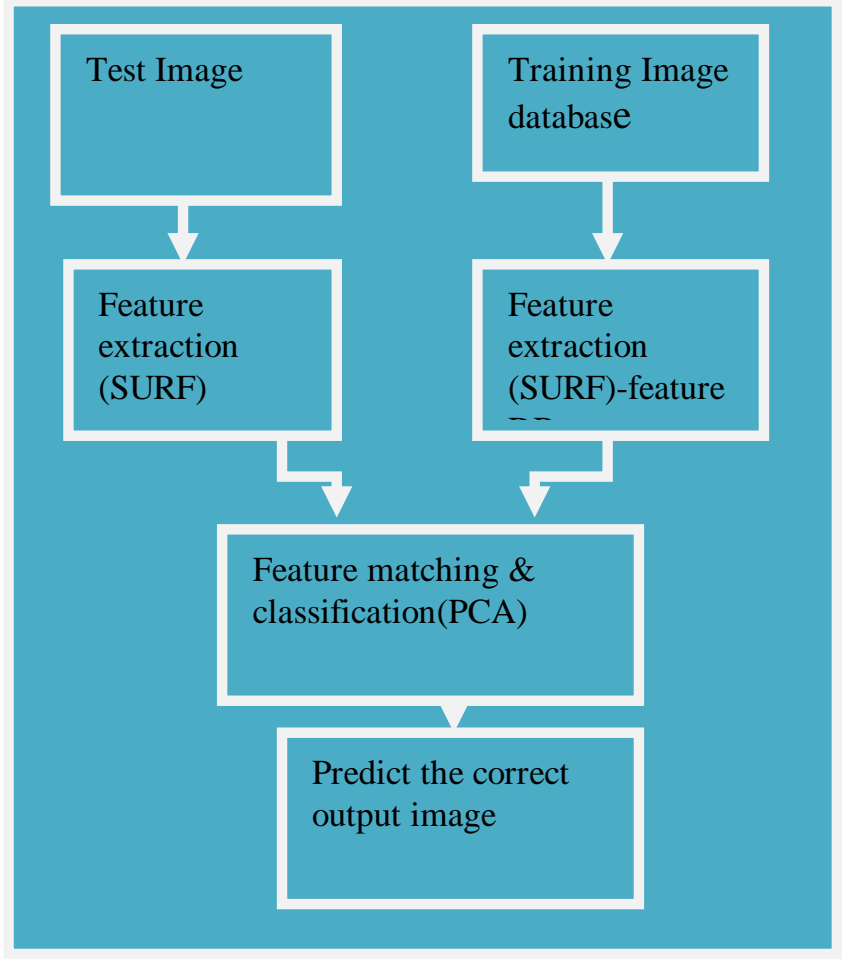

Fig 1: Framework CBMIR using PCA with SURF

\section{A. SURF Feature Detector}

The Speeded up robust features algorithm is a scale and rotation-invariant interest point detector and descriptor which is computationally very fast. It uses Integral images to improve the speed. The key points are detected by using a Fast-Hessian matrix. Computationally surf gives a very quick performance than SIFT.

\section{B. PCA Classification}

For feature selection PCA [4] has to be used as a classification method. PCA involves a mathematical procedure that transforms a number of correlated variables into a (smaller) number of uncorrelated variables called principal components. The main objective of the PCA is to reduce the dimensionality of the data set and to identify new meaningful underlying variables.

\section{Proposed Methodology}

In the proposed method, the combination of PCA with SURF feature detector is used as a CBMIR system. In which using SURF to extract the related features for the test image from the training image databases. Then extracted feature vector is classified by principal component analysis algorithm. Finally we detect the correct image set which are all related to the test image content.

\section{EXPERIMENT AND RESULTS}

In this part, we evaluate our proposed approach based on performance measures. The test data consists of 777 images belonging to 18 categories obtained from the University of Washington's object and concept recognition for CBIR research project image dataset. Each category contained varying number of images. All the images contained a textual description mentioning the salient foreground objects. The images were clustered using the algorithm with the centroids chosen at random. The cluster whose centroid was closest in distance to the given test image was determined and the images belonging to the cluster were retrieved. 


\section{Retrieved Image}

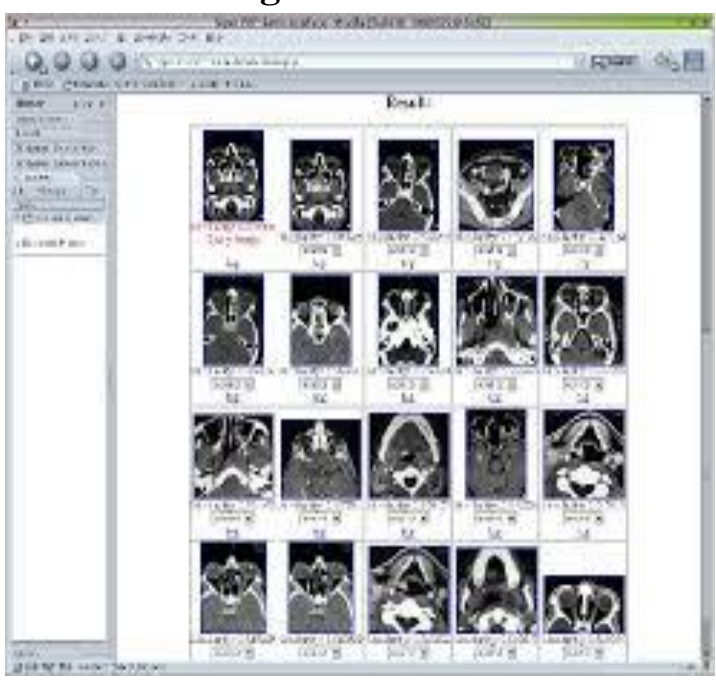

\section{Input Image}

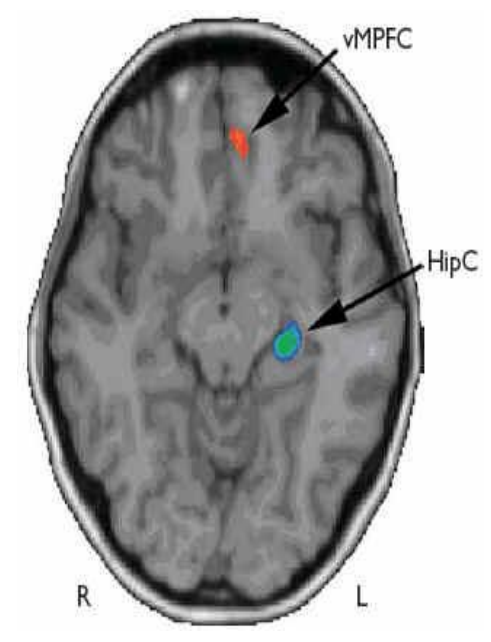

\section{CONCLUSION}

In this research article proposed approach were evaluated and perform performance measures like precision and recall. The pixel value is identified to be inappropriate in certain rectangular area; the overall region is eliminated for additional pixel comparison. The experimental results in fast work and their entire recognition time are decreased when compared to the traditional algorithm and feature extraction.

\section{REFERENCES}

1. Müller et al, "A review of content-based image retrieval systems in medical applications - clinical benefits and future directions", International journal of medical informatics, 73(1), pp:1-23,2004.

2. Agkul et al, "Content-based image retrieval in radiology: current status and future directions", Journal of Digital Imaging, 24(2), 208-222,2011.

3. Bajwal et al, "Feature Based Image classification by using Principal component analysis," CIST-Journal of Graphics, Vision and image processing, 2009

4. Sharma et al, "Face Identification Using Wavelet Transform \& PCA", Proc. of the Intl. Conf. on Advances in Computer Science and Electronics Engineering, CSEE 2013, pp. 109-113.

5. Dr. K.Somasundaram and T.Kalaiselvi "Fully automatic brain extraction algorithm for axial T2-weighted magnetic resonance images ",
Computers in Biology and Medicine Elsevier journal volume no.40, Issue no. 10,2013

6. R. Rajesh and N. Senthilkumaran, J. Satheeshkumar, B. Shanmuga Priya, C. Thilagavathy, K. Priya, "On the Type-1 and Type-2 Fuzziness Measures for Thresholding MRI Brain Images”, pp:992-995.

7. E. Ben George and M.Karnan, "MRI Brain Image Enhancement Using Filtering Techniques", International Journal of Computer Science \& Engineering Technology (IJCSET) ISSN : 2229-3345 Vol. 3 No. 9 Sep 2012 pp:399-403.

8. Napoleon D. and Praneesh M. "Detection of Brain Tumor using Kernel Induced Possiblistic C-Means Clustering”, volume no.3, issue no.9, pp 436-438, 2013.

9. M. Brown, G. Hua, and S. Winder. Discriminative learning of local image descriptors. IEEE PAMI, 33(1):43-57, 2011.

10. M. Fazel, H. Hindi, and S. P. Boyd. A rank minimization heuristic with application to minimum order system approximation. In Proc. ACC, pages 4734-4739, 2001

11. Akakin HC, Gurcan MN. Content-Based Microscopic Image Retrieval system for multi-image queries. IEEE Transactions on Information Technology in Biomedicine. 2012 Jul; 16(4):758-69.

12. Markonis D, Baroz F, Ruiz De Castaneda RL, Boyer C, Muller H. User tests for assessing a Medical Image Retrieval system: A pilot study. Studies in Health Technology and Informatics. 2012; 192:224-8.

13. Bay H, Ess A, Tuytelaars T, Van Gool L. Speeded-up Robust Features (SURF). Computer Vision and Image Understanding. 2008 Jun; 110(3):346-59.

14. Velmurugan K, Santhosh Baboo S. Content-Based Image Retrieval using SURF and color moments. Global Journal of Computer Science and Technology. 2011 May; 11(10):1-5.

15. Spyromitros-Xioufis E, Papadopoulos S, Kompatsiaris I, Tsoumakas G, Vlahavas I. An empirical study on the combination of SURF features with VLAD vectors for image search. 2012 13th International Workshop on Image Analysis for Multimedia Interactive Services (WIAMIS); Dublin. 2012 May 23-25. p. 1-4.

16. Pang Y, Li W, Yuan Y, Pan J. Fully affine invariant SURF for image matching. Neurocomputing. 2012 Jun; 85:6-10.

17. Khan NY, McCane B, Wyvill G. SIFT and SURF perfor $\neg$ mance evaluation against various image deformations on benchmark dataset. 2011 International Conference on Digital Image Computing Techniques and Applications (DICTA); Noose, QLD. 2011 Dec 6-8. p. 501-6

\section{AUTHORS PROFILE}

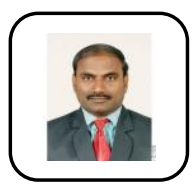

Mr. S Govindaraju, MCA, M.Phil pursed Bachelor of Commerce in the year 2002 and Master of Computer Applications from Bharathiar University, Coimbatore in the year 2005 and completed M.Phil., in Computer Science, Bharathiar University, Coimbatore in the year 2011. He is currently pursuing Ph.D., in Bharathiar University, Coimbatore since 2014 and currently working as Assistant Professor in Department of Computer Science, School of Computing, Sri Ramakrishna College of Arts \& Science (formerly S.N.R. Sons College, Coimbatore) Bharathiar University, Coimbatore, Tamil Nadu, India since 2008. He has published 6 research papers in reputed international journals and also presented research article in various conferences. His area of Interest includes Digital Image Processing and Cloud Computing. He has 11 years of teaching experience and 7 years of Research Experience.

Dr.B.Mukunthan Ph.D. pursed Bachelor of Science(Computer Science) from Bharathiar University, India in 2004 and Master of Computer Applications from Bharathiar University in year 2007 and Ph.D from Anna University-Chennai on 2013. He is currently working as Research Advisor in Department of Computer Science, Bharathidasan University, Tiruchirapalli since 2016. He is a member of IEEE \& IEEE computer society since 2009, a life member of the MISTE since 2010. He has published more than 10 research papers in reputed international journals including Thomson Reuters (SCI \& Web of Science) .He is also Microsoft Certified Solution Developer. His main research work focuses on Algorithms, Bioinformatics, Big Data Analytics, Data Mining, IoT and Neural Networks. He also invented a Novel and Efficient online Bioinformatics Tool and filed for patent. He has 11 years of teaching experience and 9 years

of Research Experience. 